\title{
Minimizing contrail formation by rerouting around dynamic ice-supersaturated regions
}

\begin{abstract}
Today, the European airspace is already faced with airspace capacity constraints, especially during business driven peak periods of the day in central European air traffic control sectors. This capacity bottleneck will cause a challenging number of severe difficulties in future flight planning and airport ground handling. Additionally, the growing public awareness and the increasing scientific knowledge of the aviation environmental impact urges air traffic stakeholders to reduce the aviation induced global warming to an acceptable level. These efforts should include both the prevention of unnecessary fuel burn due to detours and the avoidance of passing ice-supersaturated regions during cruise to prevent contrail formation. Therewith, conflicting goals have to be considered in trajectory optimization. The induced contrails influence the radiation budget of the Earth atmosphere, which depend on the dynamic size and location of the ice-supersaturated regions. However, contrail avoidance can lead to unsolvable high requirements on airspace capacity in dry and warm air spaces, where contrails are not induced.

Furthermore, contrail avoidance procedures can lead to large detours, which in turn cause more fuel burn and an increased impact on the environment due to the emission of additional radiative active substances. In this paper, the Air Traffic Control Fast Time Simulator and Air Traffic Optimizer AirTOp is used to simulate one day of Europeans air traffic and to reduce the radiative forcing of contrails by minimizing the number of flight hours through dynamic ice-supersaturated regions. Rerouting the affected flights does this, so that separation requirements are still fulfilled and each aircraft still reaches its destination. In this paper the following measures are assessed without and with contrail involved rerouting: the decrease of airspace capacity, the additional distance flown, the additional fuel burn and the contrail induced environmental impact. We found, that rerouting on this special day would have caused higher additional fuel and time costs, than saved reduced contrail costs.
\end{abstract}

Keywords: contrails, air traffic simulation, environment, efficiency, capacity
Volume 2 Issue 3 - 2018

\author{
Judith Rosenow,' Hartmut Fricke, ' Tanja \\ Luchkova, ${ }^{2}$ Michael Schultz ${ }^{2}$ \\ 'Technische Universität Dresden, Institute of Logistics and \\ Aviation, Germany \\ ${ }^{2}$ German Aerospace Center (DLR), Institute of Flight Guidance, \\ Germany
}

Correspondence: Judith Rosenow, Technische Universität Dresden, Institute of Logistics and Aviation, Dresden, Germany, Email Judith.Rosenow@tu-dresden.de

Received: March 03, 2018| Published: May 01, 2018

\section{Introduction}

Increasing the future air traffic safety, efficiency and environmental compatibility are key components of the driving air traffic research forces (NextGen) ${ }^{1}$ and (SESAR). ${ }^{2}$ These targets are becoming increasingly important in future air traffic management, considering current marked forecasts, where passenger air traffic demand is expected to grow between 4.5 percent $^{3}$ and 4.8 percent annually. ${ }^{4}$ Amongst many other important applications, safety regulations during cruise may be formulated as separation requirements affecting airspace capacity which is already a bottleneck in the air traffic system. Here, airspace capacity is defined as number of aircraft per air traffic control sector. Efficiency may also be measured by airspace capacity, controller's taskload, and by assessing the air traffic network (i.e., by considering the total distance flown within the network to serve a given demand or number of nodes). The aviation impact on the environment may be assessed by the fuel burned, which allows approximations of the total amount of the main emissions as products of complete combustion (e.g. carbon dioxide $\mathrm{CO}_{2}$ and water vapor $\left.\mathrm{H}_{2} \mathrm{O}\right) .{ }^{5}$ Approximately ten percent of all flights ${ }^{6}$ have another important impact on the environment due to the formation of condensation trails (contrails) which significantly influence the radiation budget of the Earth-atmosphere system (i.e. radiative forcing, RF). ${ }^{7}$
The avoidance of contrail formation induces large detours around or underneath ice supersaturated regions in the atmosphere,${ }^{6-9}$ which in turn cause more fuel burn and emissions and an additionally impact on efficiency and environmental compatibility itself. ${ }^{10}$ Even an impact on safety regarding separation requirements is conceivable. Furthermore, the avoidance of the dynamic ice-supersaturated regions during flight is a challenge for flight planning, because they constantly change in size, shape and location. In this case study, a twenty four hour air traffic scenario above Europe is simulated with the fast time air traffic simulator AirTOp, ${ }^{11}$ considering the dynamic influence of actually formed contrails. Beside a reference scenario without rerouting around ice-supersaturated regions, a scenario with minimum contrail radiative forcing is simulated. In the second case, a maximum number of flights are rerouted around ice-supersaturated regions at night to evaluate the number of flights, which can be rerouted without stressing safety regulations. Both scenarios are compared with respect to all mentioned key components (i.e. safety, efficiency and environment) and measures (i.e. airspace capacity, distance flown, fuel burn and the radiative forcing of the contrails). Therewith the potential of reducing the contrail radiative forcing on a normal traffic day is estimated and compared with the resulting additional flown detours, fuel burn and emissions. 
The dynamics in size, shape, global warming impact and location of contrail forming ice-supersaturated regions require the application of a 4D trajectory synchronization with a balanced weighing of computational time and expected accuracy (depending on the quality of the input parameter) such as provided by AirTOp. With a 4D trajectory simulation three coordinates in space and the time would be considered. Furthermore, AirTOp facilitates automated lateral reroutings around restricted areas, which can be individually and time variantly opened and closed. Simultaneously, AirTOp ensures the separation requirements for the complete simulated air traffic. ${ }^{11-13} \mathrm{On}$ the other hand, approximations in the aircraft performance modeling (which is limited to 2.5D due to the use of BADA performance tables) and restrictions regarding the quantification of the emissions (due to missing information of the conditions within the engine combustion chamber) have to be accepted for the benefit of computational time. A 2.5D solution space only considers arbitrary movements in the lateral direction, whereas the vertical dimension is only considered in performance tables of standard climb and descent angles for a limited number of weight-classes. Furthermore, time dependent boundary conditions cannot be considered.

The Tool chain for Multi criteria Aircraft Trajectory Optimization TOMATO, developed at TU Dresden, facilitates precise emission quantification, waypoint free trajectory optimization and rerouting, ${ }^{10}$, 14,15 but has difficulties in getting on with dynamic effects like time variant restricted areas and does not yet provide conflict detection and conflict resolution ${ }^{10,16}$ and is not suitable for the question to be answered. Furthermore, the TU Dresden Test bench for Agentbased Air Traffic Simulation (TABATS) has been developed for the trajectory synchronization for highly predictable arrivals enabled by full automation and focuses on the simulation of trajectory scenarios under realistic weather conditions (i.e. lateral rerouting around thunder cells and speed adjustments) with a specialized airport slot allocation routine. ${ }^{17-20}$ However, TABATS also concentrates on 2.5D BADA performance tables and is limited in the quantification of the emissions. In summary, considering all three key components in single air traffic flow simulation environment with satisfying precision in both the automated rerouting algorithms and the quantification of the environmental impact, requires high computational efforts and has not been done yet. In this case study, where dynamic effects of contrails are in the focus, a conventional air traffic flow simulation environment (AirTOp) has been combined with recent findings of the radiative impact of single well defined contrails, ${ }^{7,21}$ and a precise trajectory optimization is of secondary importance.

\section{Fast time simulator AirTOp}

The fast time simulator (FTS) AirTOp is a multifunctional and modular platform for the simulation of gate to gate air traffic flows considering planned 4D trajectory synchronization and negotiation, as well as airspace planned entry load and occupancy monitoring. ${ }^{11}$ Beside the parameterization of the airspace structure by Air Traffic Control (ATC) sectors, additional 3D sector blocks may be defined by polygons for lateral information and by altitude. Therewith, airspaces can be defined, consisting of different sector blocks and associated to opening schemes, i.e. different time dependent properties (e.g. restrictions, permissions or prohibitions). For closing these airspaces, different rerouting algorithms are implemented to calculate trajectories in deviation from the planned trajectories to ensure these individual user defined requirements. Therewith, ice-supersaturated regions are implemented as airspaces. Laterally time specific re-routings can be automatically computed following an envelope (dashed line in Figure 2 ) around the airspace (polygon with grey filling in Figure 2) in a distance of 2.5 nautical miles (dotted line in Figure 2). Furthermore, an interspace of 10 nautical miles is considered, before and after the envelope around the airspace, where the rerouting should start and end (compares Figure 2). Choosing the shortest path, i.e. a minimum extra distance, performs the rerouting. Furthermore, AirTOp offers further possibilities by respecting the original sector sequence or the original ATC sequence, as well as by rerouting fixed clockwise or fixed counterclockwise. Conflict resolution is iteratively realized right after the rerouting considering the whole air traffic flow until a sophisticated solution is found.

\section{Base data}

Traffic data are provided by EUROCONTROL as twenty four hour historic traffic data from 2016, July, $25^{\text {th }}$, including a flight plan of all air traffic above Europe, where 4D trajectories are updated with radar data. The data are compressed as SO6 $\mathrm{m} 3$ file. This flight plan is provided by the Network Manager Operations Centre (NMOC) to the PRISME database (i.e., a EUTOCONTROL worldwide fleet aircraft database) and processed by the Data Demand Repository (DDR2). With this data, a normal traffic day with $\approx 28000$ flights above Europe is chosen, excluding helicopters and training flights. According to the airspace structure on that day, different airspace types (deciding on prohibition, permission and conditions of entering the airspace sector) and 625 ATC sectors are modeled by using EUROCONTROL's DDR2 data base. For the benefit of an acceptable computational time, no specific weather data are implemented. Atmospheric state parameters (temperature, pressure and density) are taken from the International Standard Atmosphere (ISA). We assume, that the SO6 $\mathrm{m} 3$ reference trajectories are laterally optimized, which results in a coarse consideration of the weather conditions, at least in the reference scenario. Only for the identification of the ice-supersaturated regions, Grib2 weather data with a spatial resolution of 0.25 degrees and a time resolution of six hours, provided by the National Oceanic and Administration NOAA ${ }^{22}$ from 2016, July, $25^{\text {th }}$ is selected. The weather data are linearly interpolated for each hour, determining the change of ice-supersaturated regions in size and location over time. On that day, a typical situation in summer in the Northern hemisphere ${ }^{23}$ with relatively fast moving and small ice supersaturated regions is reflected, offering possibilities of rerouting. Furthermore, a typical movement of the ice-supersaturated regions from North to South-East due to the global circulation distracted by the Coriolis force ${ }^{24}$ has been identified on that day.

\section{Scenarios}

For the estimation of the impact of minimizing contrail formation by rerouting on safety, efficiency and environment, two air traffic scenarios have been simulated. Hereby, a reference air traffic scenario (solid great circle line in Figure 1) is compared with an air traffic scenario containing minimum contrails and maximum possible rerouting options (Figure 1, long dashed line). The parameters of both scenarios which are compared in the post simulation analysis are the following: airspace capacity (i.e. the number of aircraft per sector and per time), fuel burn [kg], additional distance flown [NM] and contrail induced environmental impact $\left[\mathrm{Wm}^{-2}\right]$ within the ice supersaturated regions. Only within the ice-supersaturated airspaces the respective parameters are compared to show the case study specific potential of 
minimizing contrail formation, hampering the $\mathrm{CO}_{2}$ emissions induced environmental impact and its consequences for airline costs. With this post analysis, statements of all key components are possible.

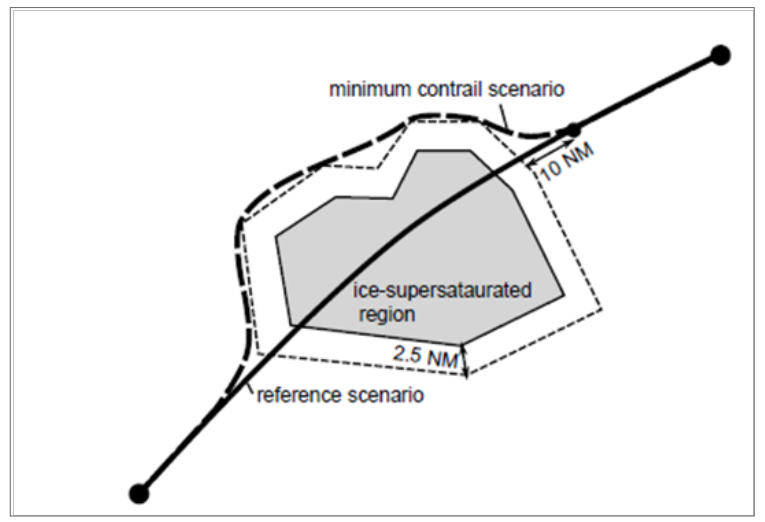

Figure I Rerouting conditions (dashed) around ice-supersaturated regions (dotted) and comparison to originally planned trajectory (line).

\section{Identification, size and location of dynamic ice-} supersaturated regions

For the estimation of the polygons describing the icesupersaturated regions, values of relative humidity over water $\mathrm{rh}_{\text {water }}$, provided by the weather data, are converted into values of relative humidity over ice $\mathrm{rh}_{\mathrm{ice}}$ according to ${ }^{7}$ and filtered by the criterion $\mathrm{rh}_{\mathrm{ice}} \geq 1$, resulting in a binary image. Afterwards, polygons are defined by connected grid points of each color of the binary image by using the polygonize tool, provided by the Geospatial Data Abstraction Library (GDAL). ${ }^{25}$ Figure 5 gives an impression on size and location of the ice-supersaturated regions. Over the whole day, there are 99 icesupersaturated regions allocated to eight pressure levels between 260 hPa (FL 320) and $190 \mathrm{hPa}$ (FL 400) with constant increments. Due to a reduced thermodynamically activity and circulation of air masses in the tropopause (the boundary layer between troposphere and stratosphere), the occurrence probability of ice-supersaturated regions is maximum at these altitudes at pressure level $\mathrm{p}=230 \mathrm{hPa}$ (FL 350) (compare Figure 2). On average, 26 ice-supersaturated regions are laterally distributed over Europe at one time step (six hours). Overall, the size of the ice-supersaturated regions is increasing with altitude (available for air traffic), which is why overflying those regions is out of question.

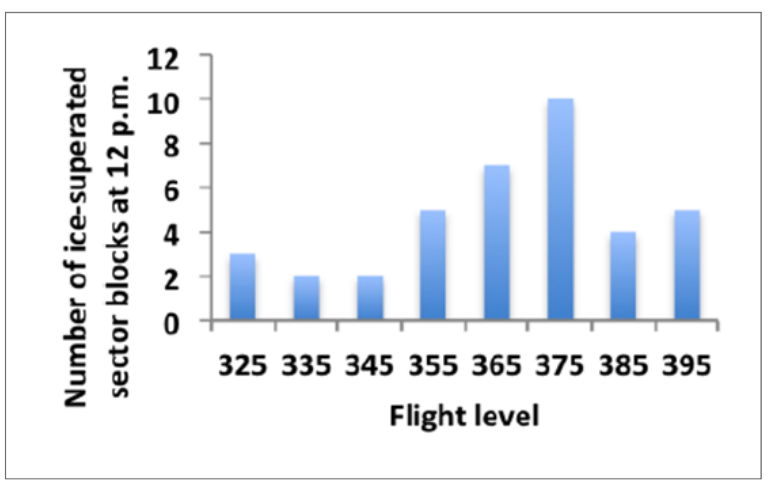

Figure 2 Number of ice-supersaturated regions per flight level at 12 p.m over Europe. The most frequently used en-route flight levels (FL 340 and FL 350) contain the most ice-supersaturated regions, due to thermo dynamical activity in the upper troposphere and lower stratosphere.

\section{Radiative forcing of contrails depending on daytime}

The environmental impact of contrails can be measured as radiative forcing RF $\left[\mathrm{Wm}^{-2}\right]$, which is the imbalance of the Earth-atmosphere energy budget due to a perturbation ${ }^{26}$ (i.e. the contrail) and depends microphysical and optical properties of the contrail as well as on the atmospheric conditions. The microphysical contrail properties depend on wavelength of solar and terrestrial radiation, ice particle size and shape, particle density, ice water content, contrail height and width and the evolution of these parameters during the contrail life cycle. ${ }^{7}$ The optical properties, on the other hand, depend on the microphysical properties and describe the particle and wavelength specific single scattering and absorption properties. ${ }^{7,27,28}$ The atmospheric conditions are covered by the specific radiation budget of the atmosphere at the actual location of the contrail, i.e. longitude, latitude, altitude, time of the day and day of the year. From this follows, the position of the sun relative to the spatial orientation of the contrail must be considered. ${ }^{7}$ This relationship can be described by the time of the day and by the aircraft heading (i.e. the flight path). For this study, the optical properties are averaged over the contrail life time according to Rosenow., ${ }^{7}$ in order to take account for the computational effort. Therewith, our model only distinguishes between contrail life time, time of the day and the duration of contrail formation. Considering the lifetime of a single contrail, the energy forcing $\mathrm{EF}\left[\mathrm{Jm}^{-1}\right]$ per meter contrail as integral over time of the radiative forcing RF depending on time $\mathrm{t}[\mathrm{s}]$ and location $\mathrm{x}$ over it's life time $\mathrm{dt}$, weighted by the contrail width $w[\mathrm{~m}]$, should be used to evaluate the contrail induced environmental impact ${ }^{29}$

$$
E F=\int_{\text {lifetime }} R F(t, x) w(t, x) d t
$$

The imbalance of the energy budget is determined by the scattering of solar radiation, which cools the atmosphere, when solar radiation is coming from above the contrail and is backscattered into the upper hemisphere. On the other hand, contrail energy forcing is controlled by absorption of terrestrial (and solar) radiation, which always heats the system. From this follows, the contrail will always heat the atmosphere during night. During dawn, sunrise and sunset, contrails, have a large heating impact on global warming as well, because of a long way of photons traveling through the contrail and due to the strong forward scattering properties of the ice particles within the contrail, a small amount of upward scattered photons can be expected. ${ }^{7}$ During daytime, the cooling effect is expected to dominate. ${ }^{7}$ Actually, East-West orientated contrails have a more significant heating effect during sunrise and sunset. ${ }^{7}$ However, this fact is not considered in this study. For the quantification of the contrail EF VázquezNavarro et al., ${ }^{22}$ tracked contrails over their whole lifetime by using the Automatic Contrail Tracking Algorithm (ACTA) for one year and averaged the optical and microphysical contrail properties. The energy forcing for this averaged contrail with a mean lifetime of 70 min at daytime of $\mathrm{EF}_{\text {day }}=-0.342 \mathrm{GJm}^{-1}$ and at nighttime of $\mathrm{EF}_{\text {night }}=$ $0.269 \mathrm{GJm}^{-1}$ have been approximated. ${ }^{21}$ These values are taken over this study for the assessment of the environmental benefit of detours around ice-supersaturated regions. These estimations show mean values of possible contrail RF measurements and underly significant fluctuations regarding the resultant RF of contrails. That's why, contrail EF was also estimated using the averaged values of RF which correspond to only a small fraction of $10 \%$ to $20 \%$ of the total number of contrails. ${ }^{21}$ 


\section{Opening schemes of dynamic ice-supersaturated regions and rerouting algorithms}

Following, ${ }^{7}$ contrails are desirable after sunrise and before sunset between 7a.m. and 7p.m. That's why airspaces with ice-super saturation are opened during this time of the day. At night (between 7p.m. and 7a.m.), 46 ice-supersaturated sector blocks are closed, to as much aircraft as possible. In the case, that the automated lateral rerouting algorithm does not find a solution, the aircraft is allowed to pass the dedicated sector block. Therewith, the maximum number of possible re-routings is assured and the environmental impact of contrails is minimized. During daytime, ice-supersaturated airspaces are open, because of the averaged cooling effect of contrails at daytime $^{21}$ (compare Section II-D).

\section{Consequences of contrail involved rerouting}

\section{Rerouted trajectory}

The successful rerouting procedure around the defined icesupersaturated airspaces proofs the ability of AirTOp to reroute around dynamic airspaces (Figure $3 \&$ Figure 4). In the example, a B777 flight from MMMX (Mexico City International Airport) to EDDF (Frankfurt Airport, Germany) is highlighted, where two icesupersaturated airspaces are flown around to avoid contrail formation. For this procedure a large detour of 367 nautical miles (Figure 3) and consequential a delay of $45 \mathrm{~min}$ (Figure 4) has to be accepted. Therefore, contrail formation could have been completely avoided. Due to the course grid based weather data (with a spatial resolution of 0.25 degrees), the definition of the ice-supersaturated airspaces by polygons is very course and edges are restricted to approximatively 90 degrees above Central Europe. Often, only narrow air spaces are present between the ice-supersaturated layers. The combination of both facts increases the difficulty for the rerouting algorithms and rerouted lateral paths are unrealistically often out of round. Considering the vertical trajectory of the described example (Figure 4) proofs, that no ice-supersaturated region (cyan and purple rectangles) is entered during the excerpt of the flight. Due to the lateral rerouting, the step climb, which has been planned at a defined waypoint, is earlier along the time line and at a different location. Which is why, the icesupersaturated airspace is not entered. During descent, the lateral rerouting algorithm also managed to avoid airspace number 116 by flying around.

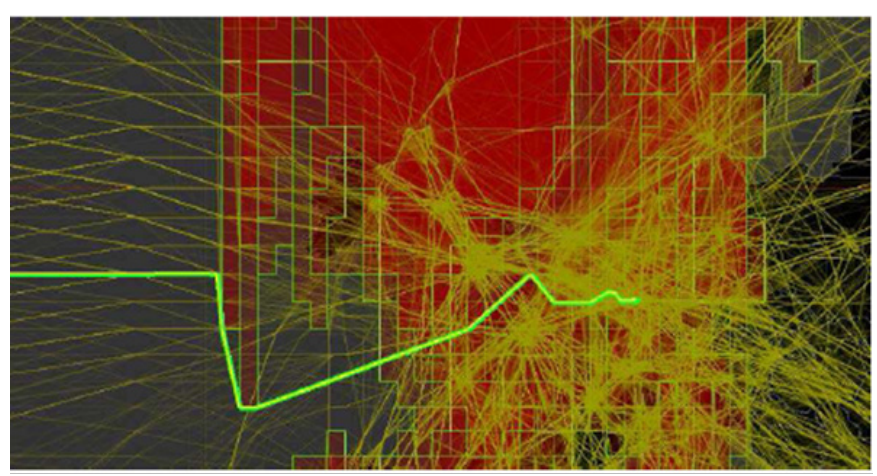

Figure 3 Excerpt of a rerouted trajectory (green) from MMMX (Mexico City International Airport) to EDDF (Frankfurt Airport, Germany) around two ice supersaturated airspaces (red) at two different flight levels. The air traffic density is indicated by the background air traffic in yellow.

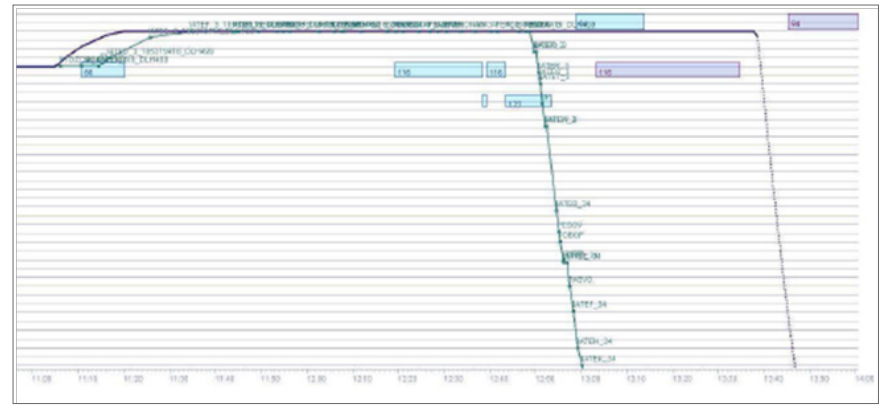

Figure 4 Excerpt of the vertical rerouted (black) versus planned (green) trajectory (the same as in Figure 3) over time (GMT). Ice-supersaturated regions are shown as cyan and purple rectangles. Due to rerouting a delay of 45 minutes has to accepted.

\section{Impact of contrail involved rerouting at night on airspace capacity}

The consequences of rerouting the air traffic around ice supersaturated regions at night on airspace capacity within the ice supersaturated regions is quantified by the number of aircraft in the respective airspaces per hour and the distance flown through these airspaces. Due to the significant number and size of ice-supersaturated regions at 12.p.m. (Figure 5), it is obvious, that not the total air traffic simulated over Central Europe can be rerouted around these airspaces. The brightness values of the yellow airspaces to be avoided in Figure 5 increase with increasing flight level. Hence, not every airspace is present at each altitude. The airspace occupancy could be reduced by 4470 aircraft at night (between 7 p.m. and 7 a.m.) across the whole European sky (Table 1). Thereby, 4.33 103 NM flight distance (and consequential contrail length) within these airspaces could have been avoided. These aircraft had been automatically rerouted considering ATC requirements, but neglecting airport slot allocation.

Table I Impact of contrail involved rerouting around Ice-supersaturated regions (here: airspace) in the upper

\begin{tabular}{|c|c|c|c|}
\hline & $\begin{array}{l}\text { Baseline } \\
\text { scenario }\end{array}$ & $\begin{array}{l}\text { Rerouting } \\
\text { scenario }\end{array}$ & Difference \\
\hline Airspace occupancy [a.u.] & $7.5110^{3}$ & $3.0410^{3}$ & $4.4710^{3}$ \\
\hline $\begin{array}{l}\text { Distance flown in airspace } \\
\text { [NM] }\end{array}$ & $9.0710^{3}$ & $1.3410^{4}$ & $4.3310^{3}$ \\
\hline Fuel burn in airspace [kg] & $4.3610^{4}$ & $1.610^{5}$ & $1.1610^{5}$ \\
\hline $\begin{array}{l}\text { Duration in airspace } \\
\text { [hh:mm] }\end{array}$ & $20: 14$ & $4: 29$ & $15: 45$ \\
\hline Contrail Energy Forcing [G]] & $3.8510^{7}$ & $1.2610^{7}$ & $2.5910^{7}$ \\
\hline Total fuel burn $[\mathrm{kg}]$ & $7.5910^{7}$ & $1.8310^{8}$ & $2.1310^{7}$ \\
\hline Total time of flight $[\mathrm{h}]$ & $1.5410^{5}$ & $3.0710^{5}$ & $2.1310^{4}$ \\
\hline
\end{tabular}

Airspace at night on total Fuel burn, total time of flight and contrail energy forcing. Due to the reduction of the number of aircraft in the Airspaces, 6.7I $10^{7}$ Kilograms additional fuel had been burned during 1.53105 Additional hours flight time for the benefit of 2.59107 Gigajoules less contrail energy forcing. 


\section{Impact of contrail involved rerouting on contrail Energy Forcing}

The rerouting results in a reduced contrail Energy Forcing of $\mathrm{EF}=2.5910^{7} \mathrm{GJ}(\approx 33 \%$ from the baseline scenario, Table 1), because $4.3310^{3} \mathrm{NM}$ contrail length could have been avoided. Compared to an average amount of incoming solar radiation of

$$
\mathrm{S}_{0}=1367 \mathrm{~J}\left(\mathrm{~s}^{-1} \mathrm{~m}^{-2}\right)
$$

${ }^{24}$ which is $\mathrm{S}=1.2110^{12} \mathrm{GJ}$ during 86400 s on an European area of $10.1810^{12} \mathrm{~m}^{2}$ and outgoing terrestrial radiation of

$$
\mathrm{F}_{0}=350 \mathrm{~J}\left(\mathrm{~s}^{-1} \mathrm{~m}^{-2}\right)
$$

${ }^{24}$ yielding $\mathrm{F}=3.0710^{11} \mathrm{GJ}$ per day (86400s) from the European land surface of $10.1810^{12} \mathrm{~m}^{2}$ (compare Figure 6), the total number of contrails enhanced the Earth-atmosphere energy budget by $2.5910^{7} \mathrm{GJ}$, which is $0.0025 \%$ of the incoming solar radiation per day (compare for a detailed derivation). Concerning a single day and an air traffic scenario reduced to Europe, this might be a negligible contribution to global warming. Transferring this data to a long time frame and the world's air traffic, the effect of aviation environmental compatibility becomes significant and rerouting around ice-supersaturated regions noteworthy.

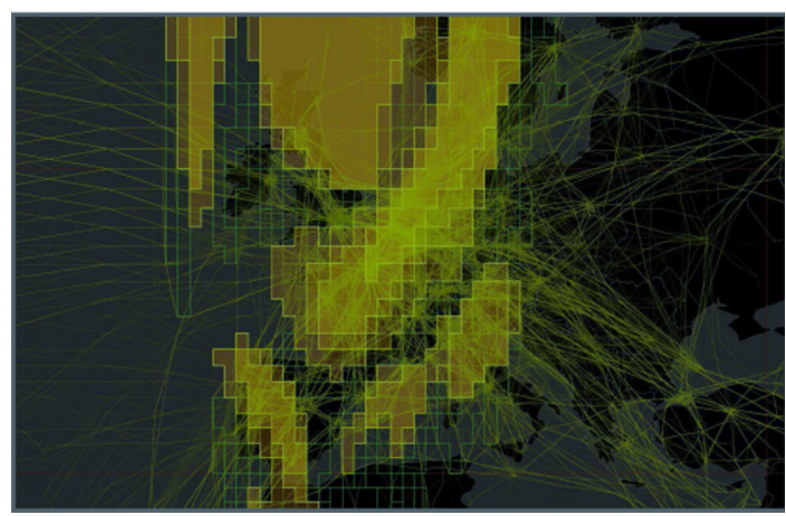

Figure 5 Simulated rerouted air traffic over Central Europe and the total number of ice-supersaturated airspaces (yellow). Due to the high air traffic density and the significant size of the airspaces to be avoided, not all aircraft could be rerouted.

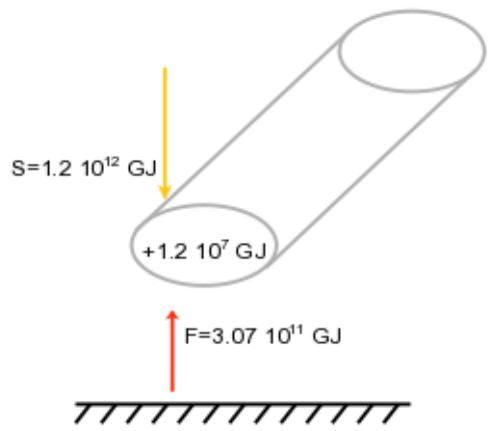

Figure 6 Relevance of European's approximated total contrail Energy Forcing, as measure of the imbalance of incoming solar radiation $\mathrm{S}$ and outgoing terrestrial radiation $\mathrm{F}$ per day. The total number of simulated induced contrails are contributing $\mathrm{EF}=3.8510^{7} \mathrm{GJ}$ to global warming. This value could be reduced by $\mathrm{EF}=2.59 \mathrm{I} 07 \mathrm{G}$ ) due to rerouting (compare Table I).

\section{Cost benefit analysis}

From an economical point of view, the rerouting cause's high operational and time dependent costs for airlines, this must be opposed to the environmental benefit. With respect to efficiency, the additional fuel burn due to the detours amounts $2.1310^{7} \mathrm{~kg}$ (Table 1). Assuming a fuel price of 0.502 euros per kilogram, ${ }^{30}$ the additional fuel burn results in $3.3610^{7}$ euros per day, which must be overcome by airlines. In other words, fuel costs increased by $46 \%$, compared to the baseline scenario. Furthermore, time costs could be considered. Approximations of mean time costs per hour of 5759euros for a medium range flown by an Airbus A320 aircraft ${ }^{16}$ allow a course order of magnitude estimate of $\approx 10^{8}$ euros extra time costs. However, this value must be treated carefully, because time costs strongly depend on flight distance, air traffic demand and network structure. Following the approximations by Rosenow et al., ${ }^{15}$ contrails induce a radiative forcing of $\approx 32$ tons of $\mathrm{CO}_{2}$ equivalent emissions per hour, which can be converted into costs by the European Emission Trading System (ETS). Assuming a price of 65 euros per ton $\mathrm{CO}_{2} \cdot{ }^{15}$ This approximation results in a reduction of 504 tons contrail induced $\mathrm{CO}_{2}$ equivalent emissions, corresponding to $3.2710^{4}$ euros.

Furthermore, engine $\mathrm{CO}_{2}$ emissions can be considered in the cost benefit analysis. Carbon dioxide is a product of complete combustion and can be quantified as linear function of fuel flow. Thereby, the small amount of carbon monoxide $\mathrm{CO}$ emissions is neglected, which must be subtracted. An emission index of 3.14 tons $\mathrm{CO}_{2}$ per ton burned fuel is assumed ${ }^{5}$ resulting in $2.3810^{6}$ and5.74 $10^{5}$ tons $\mathrm{CO}_{2}$ emissions for the baseline and the rerouted scenario, respectively. Applying the European ETS, the difference of $1.810^{6}$ tons $\mathrm{CO}_{2}$ emissions will cause $1.1710^{8}$ euros for $\mathrm{CO}_{2}$ emissions due to the detours. Hence, in this case study, if time costs are disregarded, it will be cheaper for airlines to accept the burdened contrail costs, than considering rerouting. Anyhow, this cost-benefit analysis postulates, that all airlines are equally burdened with environmental costs, induced by the total air traffic. Actually, airlines should only consider their own induced environmental costs. The resulting problem of who chooses which reroute regarding conflict resolution is nontrivial from a scientific point of view and will not be discussed in this paper. Table 2 summarized the results of the cost-benefit analysis.

Table 2 Summarized cost-benefit analysis of the rerouting scenario, compared to the baseline scenario. The values refer to additionally required fuel costs, additionally induced $\mathrm{CO}_{2}$ emissions and saved contrail costs as consequence of the rerouting around ice-supersaturated regions.

\begin{tabular}{ll}
\hline Costs induced due to & [euros] \\
\hline $\mathrm{CO}_{2}$ & $6.59 \quad 10^{4}$ \\
Fuel burn & $1.06 \quad 10^{5}$ \\
Contrails & $-3.27 \quad 10^{4}$ \\
Sum & $1.3910^{5}$ \\
\hline
\end{tabular}

\section{Conclusion}

In this case study, the ATC Fast Time Simulator and Air Traffic Optimizer AirTOp has been use for a rerouting of the European air traffic around dynamic ice-supersaturated regions at night to minimize contrail formation and therewith reduce the aviation environmental impact. A twenty four hour European air traffic scenario has been simulated and ice-supersaturated regions have been implemented as additional airspaces in the upper airspace, which where closed during night, when contrail radiative forcing is positive (i.e. have a warming effect). This scenario has been compared with a baseline scenario, where these airspaces where opened. We could reduce the number of aircraft, forming contrails by $59 \%$ and therewith reduce the contrail 
induced Energy Forcing by $\approx 2.5910^{7} \mathrm{GJ}(33 \%)$ resulting in 3.7 $10^{4}$ euros. Anyhow, the rerouting caused $2.1310^{7}$ kilograms additional fuel, which correspond to $1.0610^{7}$ euros. Even without considering time costs and other environmental induced costs (e.g. for additional $\mathrm{CO}_{2}$ emissions), the rerouting would not have been profitable on that special day, although the environmental costs are weighted very high with 65 euros per ton $\mathrm{CO}_{2}$ equivalent emissions. For the quantification of the contrail radiative forcing, the mean cooling effect of contrails during daytime has been additionally considered in both scenarios. With this case study we could show, that ice-supersaturated regions might be very large and frequent over the European airspace and lateral rerouting is not always the optimum procedure to avoid those regions, because the additional fuel burn causes higher costs than the saved environmental benefit. Anyhow, vertical rerouting is not automatically possible with AirTOp. Sometimes, unrealistic long detours and delays have been simulated, e.g. due to the rough structure of the ice-supersaturated regions, given by the weather data. However, the study is only a case study, reflecting a special weather and traffic situation. Restrictive assumptions in the modeling of contrails, in simulating re-routings and regarding the cost functions in the cost-benefit analysis had been made. The results should not be generalized so far.

\section{Outlook}

However, the possibility of considering time variant and space dependent airspace closures in a simulation environment will be used for more applications. After reducing the considered time frame to reduce the number of aircraft, a manual vertical adjustment of the trajectories will be applied, gaining more realistic trajectories, which still avoid contrail formation. Further work will be done in more precisely and detailed estimating the airline costs and the environmental costs gaining a more realistic cost-benefit analysis. In a second step, the controller's taskload will be considered in the cost analysis, which is expected to increase significantly due to the rerouting. Of further importance is the consideration of the airport slot allocation, which has been neglected in this case study, as well as real weather data and therewith a wind optimized rerouting. High computational effort is expected for an additionally planned study, wherein the timely resolution of the dynamics of the ice-supersaturated regions will be improved significantly. Two sensitivity studies are planned, first by reducing the share of ice-supersaturated areas over Europe, and second by increasing the space between the ice-supersaturated areas to determine a reference weather scenario (with respect to icesupersaturation), where rerouting should be aspired, because it will save additional environmental costs due to contrail formation.

\section{Acknowledgements}

The authors would like to thank Stanley Förster for extracting the polygons of ice supersaturated regions.

\section{Conflict of interest}

The authors declare that there is no conflict of interest.

\section{References}

1. Nextgen implementation plan 2016. USA: Federal Aviation Administration; 2016. 86 p.

2. SESAR Consortium. The roadmap for sustainable air traffic managementeuropean atm master plan. EUROCONTROL. 2012:2.
3. Global market forecast 2016-2035. Airbus Commercial Aircraft; 2016. 27 p.

4. Current market outlook 2016-2035. Boeing; 2016. 54 p.

5. Lee DS, Pitari G, Grewe V, et al. Transport impacts on atmosphere and climate: Aviation. Atmospheric Environment. 2010;44(37):4678-4734.

6. Spichtinger P. Ice oversaturated regions. Germany: University of Munich; 2004. 219 p

7. Rosenow J. Optical properties of condenstation trails. Ph.D. dissertation, Germany: Technische Universität Dresden; 2016. 189 p.

8. Sussmann R, Gierens KM. Differences in early contrail evolution of twoengine versus four-engine aircraft: Lidar measurements and numerical simulations. Journal of Geophysical Research. 2001;106(D5):4899-4911.

9. Kaiser M, Rosenow J, Fricke H, et al. Tradeoff between optimum altitude and contrail layer to ensure maximum ecological en-route performance using the enhanced trajectory prediction model (etpm). London: $2^{\text {nd }}$ International Conference on Application and Theory of Automation in Command and Control Systems (ATACCS); 2012. 8 p.

10. Rosenow J, Förster S, Lindner M, et al. Multi-objective trajectory optimization. International Transportation. 2016:Special Edition 1.

11. Airtopsoft, Belgium; 2017.

12. Kreuz M, Luchkova T, Schultz M. Effect of restricted airspace on the atm system. China: WTCR Conference; 2016. 10-15 p.

13. Luchkova T, Vujasinovic R, Lau A, et al. Analysis of impacts an eruption of volcano Stromboli could have on european air traffic. Portugal: $11^{\text {th }}$ USA/ Europe Air Traffic Management Research and Development Seminar; 2015. 19 p.

14. Luchkova T, Kaltenhaeuser S, Morlang F. Air traffic impact analysis design for a suborbital point-to-point passenger transport concept. $3^{\text {rd }}$ Annual Space Traffic Management Conference. Emerging Dynamics; 2016.

15. Rosenow J, Fricke H. Flight performance modeling to optimize trajectories. Deutscher Luft- und Raumfahrtkongress. 2016;420127:1-8.

16. Rosenow J, Förster S, Fricke H. Continuous climb operations with minimum fuel burn. Europe: $6^{\text {th }}$ SESAR Innovation days; 2016. 6 p.

17. Förster S, Rosenow J, Lindner M, et al. A toolchain for optimizing trajectories under real weather conditions and realistic flight performance. Brussels: Greener Aviation; 2016.

18. Schultz M, Fricke H, Kaiser M, et al. Universal trajectory synchronization for highly predictable arrivals. Europe: SESAR Innovation Days (SID); 2011. 18 p.

19. Schultz M, Fricke H, Kunze T, et al. Uncertainty handling and trajectory synchronization for the automated arrival management. Europe: $2^{\text {nd }}$ SESAR Innovation Days (SID); 2012.8 p.

20. Kaiser M, Schultz M, Fricke H. Automated $4 d$ descent path optimization using the enhanced trajectory prediction model (ejpm). In Proceedings of the $5^{\text {th }}$ International Conference on Research in Air Transportation (ICRAT), Berkeley: University of california; 2012.

21. Schultz M, Fricke H, Gerbothe T, et al. Modeling and evaluation of automated arrival management considering ait traffic demands. Europe: $3^{\text {rd }}$ SESAR Innovation Days (SID); 2013.9 p.

22. Vázquez-Navarro M, Mannstein H, Kox S. Contrail life cycle and properties from one year of $\mathrm{msg} / \mathrm{seviri}$ rapid-scan images. Atmospheric Chemistry and Physics Discussions. 2015;15(15):7019-7055.

23. Spichtinger P, Gierens KM, Read W. The global distribution of icesupersaturated regions as seen by the microwave limb sounder. Quarterly Journal of the Royal Meteorological Society. 2003;129(595):3391-3410. 
24. Kraus H. The atmosphere of the earth. Berlin: Springer-Verlag; 2001.

25. Schernthanner H, Bellack N. A gentle introduction to gdal/ogr: Never used the command line? no problem!. Berlin; 2017.

26. Myhre G, Shindell D, Breon FM, et al. Anthropogenic and natural radiative forcing. In: Climate change 2013: The physical science basis. Contribution of working group 1 to the fifth assessment. Report of the intergovernmental panel on climate change. UK: Cambridge University Press; 2013. 82 p.

27. Yang $\mathrm{P}$, Wei H, Huang HL, et al. Scattering and absorption property database for nonspherical ice particles in the near- through far-infrared spectral region. Appl Opt. 2005;44(26):5512-5523.
28. Hong G, Feng Q, Yang P, et al. Optical properties of ice particles in young contrails. Journal of Quantitative Spectroscopy and Radiative Transfer. 2008;109(15):2635-2647.

29. Schumann U, Graf K, Mannstein H, et al. Contrails: Visible aviation induced climate impact. In: Schumann U editors. Atmospheric physics. Berlin: Springer-Verlag; 2012. 877 p.

30. Korteland M, Faber J. Estimated revenues of VAT and fuel tax on aviation. CE Delft: Committed to the Environment; 2013. 